\title{
Desarrollo galénico y eficacia clínica de ivermectina en sistemas semisólidos para uso oral en equinos
}

\author{
Lozina, L.; Barbieri, F.; Río, F.; Bogado, E.; Ríos, E. \\ Depto. Clínicas, Facultad Ciencias Veterinarias, Univ. Nac. del Nordeste, \\ Sargento Cabral 2139, Corrientes, Argentina. E-mail: 1lozina@vet.unne.edu.ar
}

\begin{abstract}
Resumen
Lozina, L.; Barbieri, F.; Río, F.; Bogado, E.; Ríos, E.: Desarrollo galénico y eficacia clínica de ivermectina en sistemas semisólidos para uso oral en equinos. Rev. Vet. 29: 2, 93-97, 2018. La ivermectina (IVM) es un compuesto prácticamente insoluble en agua y fácilmente soluble en solventes orgánicos. Su distribución en el organismo es buena pero la absorción oral es variable, siendo ésta una condición limitante de la biodisponibilidad. La solubilidad y la liberación de estos compuestos se puede mejorar usando vehículos que posean propiedades autoemulsionantes como la carboximetilcelulosa sódica (CMCNa) y los poloxámeros (P407). El objetivo del presente trabajo fue desarrollar formulaciones eficaces y seguras con CMCNa y P407 para el control de parásitos gastrointestinales en equinos y evaluar su eficacia clínica. Se trabajó con 33 equinos, algunos puros y otros mestizos de la raza Silla Argentino, con condición corporal 4-5 y aspecto general regular a bueno. Se extrajeron muestras de materia fecal, el día 0 y posteriormente se administraron los tratamientos antiparasitarios antes mencionados. Para determinar la eficacia de los productos se tomaron nuevas muestras los días 3,30 y 60 , efectuándose el test de reducción de conteo de huevos por gramo de materia fecal (HPG). Los resultados del primer muestreo fueron positivos a nematodes y negativo a cestodes; el recuento HPG osciló entre 20 y 1690. Luego de los tratamientos no se observaron efectos adversos en los animales, lo cual significa que los excipientes utilizados son seguros. Ambas formulaciones fueron efectivas en la reducción del conteo de huevos, pero de manera diferente. Se concluye que ambos tratamientos fueron eficaces, aunque P407 resultó más adecuado como sistema de liberación sostenida del principio activo.
\end{abstract}

Palabras clave: equino, parásitos gastrointestinales, ivermectina, uso oral, eficacia.

\begin{abstract}
Lozina, L.; Barbieri, F.; Río, F.; Bogado, E.; Ríos, E.: Pharmaceutical development and clinical efficacy of ivermectin semisolid systems for oral oral administration in horses. Rev. Vet. 29: 2, 93-97, 2018. Ivermectin (IVM) is a compound insoluble in water and easily soluble in organic solvents. Its distribution in the body is good and oral absorption is variable, this condition is a limitation of its bioavailability. The solubility and release of IVM can be improved by using self-emulsifying vehicles such as sodium carboxymethylcellulose and poloxamers. Aims of this work were to develop effective, safe formulations for the control of horse gastrointestinal parasites and to evaluate their clinical efficacy. We worked with 33 horses, some were pure breed while others were half-breed Silla Argentino, with 4-5 body condition and a fair to good general condition. On day 0 stool samples were collected and treatment with the formulations under study were administered. On days 3, 30 and 60 stool samples were collected to determine the reduction in eggs per gram of feces (EPG). The initial stool test was positive for roundworms and negative for tapeworms, the EPG ranged from 20 to 1690. After deworming, no adverse effects were observed in animals, demonstrating this the safeness of excipients used in our preparations. Both formulations were effective in reducing egg count, but in different ways. We conclude that both treatments were successful, however treatment 2 proved to be a more suitable sustained release system of the active ingredient.
\end{abstract}

Key words: horse, gastrointestinal parasites, ivermectin, oral use, efficacy. 


\section{INTRODUCCIÓN}

El equino constituye una herramienta de trabajo de mucha importancia para el hombre. Un gran número de productores se dedica a la cría de equinos para deporte $\mathrm{y}$ tareas rurales, donde el rendimiento está condicionado a su estado de salud.

En el nordeste argentino son frecuentes las parasitosis gastrointestinales, que generalmente conviven con el animal y se presentan de forma sub-clínica, provocando pérdidas en el desarrollo corporal y disminución en el rendimiento de los animales ${ }^{9}$. Los parásitos gastrointestinales más frecuentes son helmintos de las familias Strongylidae, Ascarididae, Oxyuridae y Anoplocephalidae ${ }^{12}$.

La ivermectina (IVM) es un antiparasitario de amplio espectro, eficaz contra una gran variedad de nematodes y ectoparásitos, pero sin acción contra cestodes ni trematodes. En equinos, es común utilizar la vía oral para administrar el producto en forma de gel o pasta y no son recomendables otras vías como IM o SC, dado que pueden desarrollarse lesiones sépticas, cuya inflamación impediría la dinámica de la absorción ${ }^{21}$.

La IVM pertenece a las lactonas macrocíclicas. En estado puro es un polvo de color blanco, poco o nada soluble en agua, pero sí en solventes orgánicos; muy lipofílica por lo que su distribución en el organismo es buena, presentando un elevado porcentaje de unión a proteínas plasmáticas, concentrándose en grasa e hígado $^{21}$. Se excreta principalmente por la bilis desde donde es vehiculizada hacia el intestino y eliminada bajo su forma activa a través de las heces ${ }^{16}$.

En los últimos años, se ha producido un interés creciente en el desarrollo de excipientes que mejoren las propiedades biofarmacéuticas de los fármacos de administración oral, utilizando sustancias que puedan formar sistemas semisólidos que aumenten la solubilidad del principio activo y resulten adecuados como sistemas de liberación sostenida del mismo.

Los objetivos propuestos en el presente trabajo fueron desarrollar formulaciones orales eficaces y seguras para el control de parásitos gastrointestinales en equinos.

\section{MATERIAL Y MÉTODOS}

Desarrollo de las formulaciones. Se realizaron ensayos de solubilidad de IVM usando diferentes mezclas de alcohol etílico, bencílico, propilenglicol, polisorbato 80 y polisorbato 20 . A partir de los resultados se seleccionó como disolvente aquel que logró obtener el principio activo (al 1\%) en una solución estable, a una concentración del $10 \%$ de la mezcla final. Se utilizaron dos excipientes para incorporar la solución del principio activo, carboximetilcelulosa sódica $(\mathrm{CMCNa})$ al $5 \%(\mathrm{~T} 1)$, disuelta mediante agitación en agua destilada a temperatura ambiente y poloxamero (P407) al $40 \%$ (T2), mediante el método frío, adicionando lentamente agua destilada a $5^{\circ} \mathrm{C}$ bajo agitación constante.
La dispersión se mantuvo en el refrigerador (6-12 h) hasta lograr la disolución total. Las concentraciones se expresaron como porcentaje peso en peso $(\% \mathrm{p} / \mathrm{p})$. Una vez obtenidas ambas formulaciones se les adicionó un saborizante para mejorar su palatabilidad.

Para el estudio de estabilidad física a corto plazo, las formulaciones fueron almacenadas durante 30 días a $25 \pm 2^{\circ} \mathrm{C}$ y $70 \% \pm 5 \%$ de humedad relativa, evaluadas cada 7 días, así como también, realizando ensayos de centrifugación, usando $2 \mathrm{~g}$ de la formulación, centrifugados durante $15 \mathrm{~min}$ a $3000 \mathrm{rpm}$, seguida de la observación de las características organolépticas y mediciones de $\mathrm{pH}$.

Pruebas de eficacia clínica. Se trabajó con 33 equinos de diferente sexo y condición fisiológica, con edades comprendidas entre los 7 meses y 27 años, con condición corporal y aspecto general regular a bueno; con signos clínicos compatibles con parasitosis gastrointestinal. Posteriormente se extrajeron muestras de materia fecal a partir del recto para realizar el conteo de huevos por gramo (HPG) inicial.

El análisis de sangre comprendió las determinaciones de hematocrito (Hto), recuento de glóbulos rojos y concentración de hemoglobina ( $\mathrm{Hb})$. Los animales fueron pesados para el posterior cálculo de dosis utilizando una balanza electrónica portátil; luego se dividió el lote en tres grupos homogéneos de acuerdo al número de HPG que arrojó el análisis inicial. Así, once animales conformaron el grupo T1, otros once el grupo T2 y los once restantes el grupo control (placebo).

El día 0, cada grupo recibió el tratamiento correspondiente, a razón de $2 \mathrm{~g} / 100 \mathrm{~kg}$ de peso vivo (equivalente a $0,2 \mathrm{mg} / \mathrm{kg}$ del principio activo). Los geles se depositaron sobre la base de la lengua con una jeringa plástica e inmediatamente se elevó la cabeza del animal para favorecer la deglución. A los 3, 30 y 60 días se tomaron nuevamente muestras de heces fecales para determinar HPG, a fin de evaluar la eficacia de los tratamientos.

Observaciones clínicas luego de los tratamientos. Los animales tratados fueron monitoreados a las 2, 4, 24 y $48 \mathrm{~h}$ post tratamiento para determinar la posibilidad de efectos adversos. Las observaciones incluyeron aspectos clínicos generales, tales como estado de excitación o depresión del sensorio, alteraciones de la locomoción, presencia de diarrea o constipación y signos de cólicos.

Análisis estadístico. El análisis de los datos fue realizado utilizando el programa Infostat/profesional versión 2006. Los resultados arrojados por la estadística descriptiva fueron presentados en tablas conteniendo la media aritmética y el desvío estándar de HPG en los diferentes días, así como los valores de Hto y $\mathrm{Hb}$ que presentaron los equinos. La eficacia de los tratamientos fue evaluada utilizando la fórmula de Abbott ${ }^{1}$ 
y representada en porcentaje de eficacia y medias del conteo de la reducción de HPG en los días analizados.

\section{RESULTADOS Y DISCUSIÓN}

Desarrollo de la formulación. Se pudo observar una adecuada disolución del principio activo utilizando etanol de $96^{\circ}$. La disolución en el resto de los disolventes no fue adecuada en las concentraciones utilizadas. La incorporación a los sistemas semisólidos dio como resultado geles de características homogéneas, consistencia adecuada y sin separación de fases ni presencia de partículas en suspensión, lo que resulta sumamente importante para asegurar su correcta administración y liberación de la droga del sistema portador. Estas características se mantuvieron estables en los ensayos de centrifugación.

Las características organolépticas no variaron en el tiempo. El pH de las formulaciones 1 y 2 fue de $7,30 \pm 0.02$ y de $7,05 \pm 0.02$, respectivamente. La biodisponibilidad oral depende de la solubilidad acuosa, lo cual se convierte en un factor limitante para fármacos liposolubles debido a la mala disolución en los fluidos gastrointestinales. Por lo tanto, la disolución in vitro ha sido reconocida como un paso fundamental en el desarrollo de dichos fármacos ${ }^{17}$.

Ciertos estudios evidenciaron que la velocidad de disolución se puede mejorar usando vehículos que posean actividad superficial o propiedades autoemulsionantes ${ }^{4}$. Dos excipientes fueron utilizados para desarrollar el sistema semisólido conteniendo IVM: CMCNa y poloxámero 407.

La primera, por sus propiedades mucoadhesivas, fue adecuada para la administración oral de los geles, observándose una total deglución del preparado con una consistencia óptima en la concentración utilizada del $5 \%$. Este polímero es la sal sódica del éter policarboximetílico de la celulosa, soluble en agua, coloide hidrófilo que da geles de buena consistencia pero sin una gran transparencia, con propiedades adhesivas, lo que los hace muy útiles como excipientes para sustancias semisólidas de uso oral, admitiendo la incorporación de hasta un $15-20 \%$ de alcohol ${ }^{18}$.

La propiedad del poloxámero de facilitar la incorporación de principios activos insolubles en solventes acuosos, fue considerada para la selección del polímero como vehículo para el desarrollo de la segunda formulación. Estas, fueron sustancias de características tensioactivas, formadas por la unión de bloques moleculares de polioxietileno (hidrófilo) y polioxipropileno (hidrófobo) ${ }^{4,6}$. En el presente trabajo, la concentración ideal para la formulación del gel de IVM fue del $40 \%$.

Eficacia clínica. Los resultados del hemograma evidenciaron un $52 \%$ de equinos anémicos, los que al día 0 presentaron principalmente valores bajos en los parámetros $\mathrm{Hto} \mathrm{y} \mathrm{Hb}$. El resto de los parámetros se encontró dentro del rango de valores normales. El cuadro hemático de cada animal fue controlado a través del hemograma, teniendo en cuenta como valores de referencia Hto del 32-47\%, recuento de eritrocitos de 6,410 millones/ml, y Hb de 11-17 gr/dl ${ }^{13}$.

Luego de 30 y 60 días, los grupos tratados con ambas formulaciones de IVM incrementaron estos valores, mientras que el grupo control no presentó variaciones respecto al inicio del tratamiento (Tabla 1).

En el equino, la anemia puede ser secundaria a múltiples causas. Entre las más frecuentes se hallan las producidas por el virus de la anemia infecciosa equina (que en este estudio fue descartada por el test de Coggins) y enfermedades parasitarias ${ }^{20}$. Las causadas por infestación masiva de estróngilos y/o por hemoparásitos son de importancia en esta región ${ }^{19}$, habiendo sido la segunda, descartada mediante el seguimiento de los animales por extendidos finos de sangre.

$\mathrm{El}$ incremento de valores obtenidos de $\mathrm{Hto}$ y $\mathrm{Hb}$ en los grupos tratados con IVM, sugieren que la anemia es posiblemente secundaria a la intensa parasitosis corroborada por la persistencia de estos valores en el grupo control. Luego de la administración de las formulaciones antiparasitarias no se observaron efectos adversos durante el periodo evaluado.

En general, la presencia de parásitos internos suele cursar de forma sub-clínica, provocando pérdida de peso, alteraciones en el hemograma (anemia, linfocitosis) y mal aspecto del pelaje ${ }^{8}$. Los más comunes son los nematodes y el tratamiento suele ser a base de antiparasitarios administrados por vía oral. Los endoparásitos muertos suelen verse a simple vista en las heces o mediante análisis de la materia fecal ${ }^{5}$.

En cuanto al examen coproparasitológico inicial, el cualitativo arrojó resultados positivos a nematodes y negativos a cestodes, observándose huevos compatibles con las familias Strongylidae, Ascarididae, Oxyuridae y Strongyloididae. En los análisis cuantitativos, el HPG osciló entre 20 y 1690, como indica la Tabla 2. Luego de conformar los grupos experimentales, los promedios de HPG fueron de 1664 en el Grupo 1; 2707 en el Grupo 2 y 3699 en el Grupo 3.

En la Tabla 2 se pueden observar los valores máximos y mínimos de HPG, así como el desvío estándar y el promedio que se obtuvo en los muestreos posteriores a la desparasitación de cada grupo. Evaluando los resultados obtenidos luego de la administración de

Tabla 1. Valores de los parámetros hematológicos de los equinos.

\begin{tabular}{ccccc}
\hline día & $\mathrm{P}$ & $\mathrm{G} 1$ & $\mathrm{G} 2$ & $\mathrm{G} 3$ \\
\hline 0 & $\underline{\mathrm{Hb}}$ & $9,81 \pm 1,51$ & $9,78 \pm 0,70$ & $9,20 \pm 1,10$ \\
& $\underline{\mathrm{Hto}}$ & $28,33 \pm 2,78$ & $29,33 \pm 2,12$ & $29,56 \pm 2,10$ \\
\hline 30 & $\underline{\mathrm{Hb}}$ & $13,01 \pm 0,90$ & $12,10 \pm 0,50$ & $9,30 \pm 1,15$ \\
& $\underline{\text { Hto }}$ & $35,00 \pm 3,04$ & $36,00 \pm 3,09$ & $30,78 \pm 0,87$ \\
\hline 60 & $\underline{\mathrm{Hb}}$ & $13,34 \pm 0,80$ & $12,88 \pm 0,58$ & $9,26 \pm 1,21$ \\
& $\underline{\text { Hto }}$ & $35,33 \pm 3,03$ & $36,18 \pm 3,07$ & $29,44 \pm 1,40$ \\
\hline
\end{tabular}

P: parámetro; G1: formulación con carboximetilcelulosa (CMCNa); G2: formulación con poloxamero (P407); G3: placebo. 
Tabla 2. Carga parasitaria en los diferentes grupos de equinos, antes y después del tratamiento.

\begin{tabular}{ccccc}
\hline \multirow{2}{*}{ grupo } & \multicolumn{4}{c}{ huevos por gramo de heces } \\
\cline { 2 - 5 } & día & $\overline{\mathrm{x}} \pm \mathrm{DE}$ & mínimo & máximo \\
\hline \multirow{4}{*}{1} & 0 & $664 \pm 452$ & 260 & 1470 \\
& 3 & $4 \pm 12$ & 0 & 40 \\
& 30 & $26 \pm 51$ & 0 & 150 \\
& 60 & $74 \pm 100$ & 0 & 280 \\
\hline \multirow{4}{*}{2} & 0 & $707 \pm 495$ & 30 & 1650 \\
& 3 & $20 \pm 28$ & 0 & 80 \\
& 30 & $0 \pm 0$ & 0 & 0 \\
& 60 & $5 \pm 13$ & 0 & 40 \\
\hline \multirow{3}{*}{3} & 0 & $699 \pm 652$ & 20 & 1690 \\
& 3 & $758 \pm 652$ & 40 & 1703 \\
& 30 & $891 \pm 595$ & 60 & 1725 \\
& 60 & $859 \pm 557$ & 65 & 1700 \\
\hline
\end{tabular}

$\overline{\mathrm{X}} \pm$ DE: media aritmética \pm desvío estándar

Tabla 3: Porcentaje de eficacia observado en el test de reducción del conteo de huevos a los 3, 30 y 60 días post-tratamiento.

\begin{tabular}{cccc}
\hline trat. & HPG día 3 & HPG día 30 & HPG día 60 \\
\hline 1 & $4,55(99,4 \%)$ & $26,4(97,1 \%)$ & $74(91,4 \%)$ \\
2 & $20(97,4 \%)$ & $0(100 \%)$ & $4(99,5 \%)$ \\
3 & 757,73 & 891,36 & 859,55 \\
\hline
\end{tabular}

trat.: tratamiento; HPG: huevos por gramo de heces $(\overline{\mathrm{x}})$

los tratamientos, se puede constatar que ambas formulaciones fueron eficaces en la reducción del conteo de huevos.

En el presente trabajo, la eficacia para la formulación 1 fue del $99 \%$ al día 3, nivel que luego descendió a 97 y $92 \%$ en los días 30 y 60 respectivamente. Por su parte, para la formulación 2, al día 3 se observó una eficacia de $97 \%$, que llegó al $100 \%$ al día 30 y se mantuvo en $99 \%$ al día 60 (Tabla 3). En el Grupo 3, que no recibió ningún tratamiento, se puede observar como el parámetro HPG continuó incrementándose hasta el momento de la desparasitación luego de la finalización del ensayo.

Estos datos concuerdan con lo reportado por autores que evaluaron el período de reaparición de huevos $(\mathrm{PRH})$ en equinos desparasitados con $\mathrm{IVM}^{3}$. Este período se considera como una potencial alerta temprana al desarrollo de los fenómenos de resistencia, la cual originalmente era de 9 a 13 semanas cuando la droga fue registrada en el mercado veterinario mundial. Actualmente, el PRH para IVM es de 6 a 8 semanas y valores inferiores son interpretados como resistencia emergente ${ }^{10,14} \mathrm{y}$ estarían asociados a la sobrevida de larvas ciatostomas de cuarto estadio ${ }^{10,11}$. En 2017, investigadores argentinos usaron en sus investigaciones el concepto de PRH, estableciendo que existe eficacia cuando la reducción en el conteo de huevos por gramo es mayor al $90 \%$ para las lactonas macrociclicas ${ }^{2}$. En el presente trabajo, ambas formulaciones mantuvieron la eficacia luego de 8 semanas.
Asimismo, a los 60 días ambos preparados logran mantener los valores de conteo de huevos por debajo de los 300 HPG (Tabla 3). Cabe resaltar, que nuestro equipo de investigación realizó estudios durante los últimos cinco años con los mismos equinos y esta droga reveló una eficacia mayor al $90 \%$ en todos los casos (resultados no mostrados), indicando que luego de más de 30 años de uso de esta lactona macrocíclica, no ha desarrollado resistencia, manteniendo una eficacia muy alta de acuerdo al test in vivo. Sin embargo, es importante considerar un cuidadoso programa rotacional de antiparasitarios diseñado de acuerdo a la situación particular de cada explotación, como la opción más adecuada para asegurar el control de las parasitosis gastrointestinales en concordancia con otros autores ${ }^{7}$.

Una terapia selectiva, donde se analizan las muestras fecales de todos los equinos pero sólo los que superan un valor de corte predeterminado reciben tratamiento, minimiza la contaminación de los pastos mediante la eliminación de gusanos adultos de estos parásitos, los cuales liberan la mayor proporción de huevos.

Existe evidencia científica que esta terapia selectiva disminuye en gran medida la contaminación total de los pastos y se puede esperar a continuación que los equinos se infestarán con menor intensidad. Pero al mismo tiempo, se mantiene un nivel biológicamente importante del refugio parasitario, ya que los caballos que permanecen sin tratar eliminan un bajo número de huevos ${ }^{12,15}$.

Se concluye que ambas formulaciones fueron efectivas tanto en la reducción de HPG, como en el mantenimiento de bajos niveles en el recuento de huevos durante 60 días. Así, los desarrollos mostraron ser inocuos, es decir, los animales no presentaron reacciones adversas a la administración, por lo cual constituirían una alternativa válida para el tratamiento y control de los parásitos gastrointestinales del equino.

\section{REFERENCIAS}

1. Abbott WS. 1925. A methods of comparing the effectiveness of insecticide. J Econ Entomol 18: 265-267.

2. Anziani O, Ardusso G. 2017. Resistencia a los antihelmínticos en nematodes intestinales que parasitan a los equinos en Argentina. RIA 43: 24-35.

3. Ardusso G et al. 2016. Anthelmintic resistance in equine nematodes in Argentina. J Equine Vet Sci 39: 52-53.

4. Castro SG, Bruni S, Lanusse CE, Allemandi DA, Palma DS. 2010. Improved albendazole dissolution rate in pluronic 188 solid dispersions. AAPS Pharm Sci Tech 11: 1519-1525.

5. Cros FL. 2001. Principales patologías y enfermedades del caballo. Revista Ganadería 1: 50-52

6. Freitas MN, Farah M, Bretas RE, Ricci JE, Marchetti JM. 2006. Rheological characterization of poloxamer 407 nimesulidegels. Rev Ciênc Farm Básica Apl 27: 113-118.

7. Gaudencio LF. 2005. Cría y manejo del caballo. Tesis para obtener el título de Ing. Agronomo Zootecnista, Univ. Autón.Antonio Narro, Buenavista, México, 113 p. 
8. Ionita $\mathbf{M}$ et al. 2010. Use of a reverse line blot assay to survey small strongyle (Strongylida: Cyathostominae) populations in horses before and after treatment with ivermectin. Vet Parasitol 168: 332-337.

9. Lamberti R, Gino L, Calvo C, Bertorello MG, Benito A. 2008. Epidemiología y parasitismo gastrointestinal en equinos del departamento Maracó, Provincia de La Pampa, Argentina. Ciencia Veterinaria 10: 32-36.

10. Lyons ET, Tolliver SC, Collins SS. 2011. Field tests demostrating reduced activity of ivermectin and moxidectin against small strongyles in horses on 14 farms in Central Kentucky in 2007-2009. Parasitol Res 108: 355-360.

11. Lyons ET, Tolliver SC. 2013. Further indication of lowered activity of ivermectin on immature small strongyles in the intestinal lumen of horses on a farm in Central Kentucky. Parasitol Res 112: 889-891.

12. Mendoza HD, Muñoz DL, León GJ. 2014. Helmintos gastrointestinales en equinos de tracción urbanos de países tropicales en vía de desarrollo. Rev Zoot 1: 14-23.

13. Meyer DJ, Harvey JW. 2007. Medicina laboratorial veterinaria. Interpretacion y diagnosis, 3ra. ed., Multimédica - Ediciones Veterinarias, p. 424-427.
14. Nielsen MK et al. 2013. AAEP Parasite Control Guidelines, American Association of Equine Practitioner, p. 24. https://aaep.org/sites/default/files/Guidelines/AAEP ParasiteControlGuidelines_0.pdf

15. Pérez $\mathbf{R}$ et al. 1999. Comparison of the pharmacokinetics of moxidectin (Equest $\AA)$ and ivermectin $($ Eqvalan () in horses. J Vet Pharmacol Therap 22: 174-180.

16. Pérez R, Cabezas I, Sutra JF, Galtier P, Alvinerie M. 2001. Faecal excretion profile of moxidectin and ivermectin after oral administration in horses. Vet $J$ 161: 85-92.

17. Prakash K et al. 2014. Pharmaceutical particle technologies: An approach to improve drug solubility, dissolution and bioavailability. Asian J Pharm Sci 9: 304-316.

18. Rowe RC, Sheskey PJ, Quinn ME. 2009. Handbook of Pharmaceutical Excipients, $6^{\text {th }}$ Ed., Washington, USA, p. 917.

19. Robinson EN. 1992. Terapéutica actual en medicina equina, Ed. Prensa Veterinaria Argentina, Buenos Aires, p. 320-323.

20. Reed SM, Bayly WM, Sellon DC. 2009. Equine Internal Medicine, $3^{\circ}$ ed., W.B. Saunder Co., Philadelphia, p. 211.

21. Sumano HS, Ocampo L. 2006. Farmacología veterinaria, $3^{\mathrm{a}}$ ed., McGraw-Hill Interamericana, México, p. 473474.

\section{S JR SClmago Journal \& Country Rank}

\section{Powered by SCEPUS}

\section{Revista Veterinaria mantiene su índice de impacto}

Noticias de Scimago Research Group (Scimago Journals \& Country Ranks, Scopus-Elsevier) comunican que la publicación de nuestra casa, Revista Veterinaria, aumentó su índice de impacto. El índice SJR mide la influencia científica (impacto) del artículo de una revista, expresando cuán importante es el "artículo promedio" de la publicación en la discusión científica global (sistema Thomson Reuters).

Para nuestra revista, tal indicador había sido de 0,03 entre 2008 y 2011, aumentando a 0,05 en 2012, a 0,11 en 2013, a 0,108 en 2015 y a 0,100 en 2017, último período evaluado. Asimismo, surge para nuestra publicación un sostenido descenso del indicador que relaciona "citas versus autocitas", demostrando que los autores de los artículos están abandonando la práctica de citar sus propias publicaciones anteriores.

Por último, se advierte que según este portal, nuestra "Revista Veterinaria" continúa siendo la única publicación de esta rama de la ciencia que posee índice de impacto en Argentina. Para el resto del cono sur tal distinción recae en Brasil, Chile, Colombia y Venezuela. En revistas de veterinaria, a nivel mundial el mayor índice de impacto (2,45 puntos) recae en Annual Review of Animal Biosciences (Estados Unidos). 